\title{
Mass, Distance, Spin, Charge, and Orientation of the super massive black hole SgrA*
}

\author{
Andreas Eckart*, A.A. Tursunov ${ }^{1,3}$, M. Zajaček ${ }^{2,1}$, M. Parsa ${ }^{1}$, E. Hosseini ${ }^{1,2}$, M. \\ Subroweit $^{1}$, F. Peissker ${ }^{1}$, C. Straubmeier ${ }^{1}$, M. Horrobin ${ }^{1}$, V. Karas ${ }^{4}$ \\ 1) I. Physikalisches Institut der Universität zu Köln, Zülpicher Str. 77, D-50937 Köln, Germany; \\ 2) Max-Planck-Institut für Radioastronomie, Auf dem Hügel 69, D-53121 Bonn, Germany; \\ 3) Institute of Physics and Research Centre of Theoretical Physics and Astrophysics, Silesian \\ University in Opava, Bezručovo nám.13, CZ-74601 Opava, Czech Republic; \\ 4) Astronomical Institute of the Academy of Sciences Prague, Bocni II 1401/1a, CZ-141 31 \\ Praha 4, Czech Republic \\ E-mail: eckarteph1.uni-koeln.de
}

\begin{abstract}
SgrA* at the center of the Milky Way is the supermassive black hole that is most suited for a determination of its fundamental properties. Here, we summarize the most recent results on mass and distance, spin, and charge, and put them into the context of representative previous measurements. The data which these results are based upon have mostly been taken with large infrared single telescopes, the ESO VLTI infrastructure combined with the GRAVITY interferometer, as well as the X-ray imaging using Chandra. One finds: $R_{S g r A *}=8.12 \pm 0.03 \mathrm{kpc}$ and $M_{S g r A *}=$ $(4.10 \pm 0.03) \times 10^{6} \mathrm{M}_{\odot}$, corresponding to relative uncertainties in these quantities of about $0.4 \%$ and $0.7 \%$. Spin values above 0.5 are suggested but the model dependent determination is not yet very conclusive. Models suggest that the spin vector is orientated towards the East and inclined towards the observer at an appreciable angle. An observational upper limit of $Q_{S g r A *} \leq 3 \times 10^{8} \mathrm{C}$ can be given for the charge.
\end{abstract}

Accretion Processes in Cosmic Sources - II - APCS2018

3-8 September 2018

Saint Petersburg, Russian Federation

${ }^{*}$ Speaker. 


\section{Introduction}

The supermassive black hole SgrA* at the center of the Milky Way is the closest of its kind and can be studied in unprecedented detail (Eckart \& Genzel 1996, 1997, Ghez et al. 1998, Eckart et al. 2017). Hence, its properties like mass and distance, spin, and charge - or the limits on them - can be obtained rather accurately. The position of the central black hole SgrA* can be obtained by Very Long Baseline (VLBI) measurements. Here, Menten et al. (1997) and Reid et al. (2003) find (J2000) R.A. $=17^{h} 45^{m} 40.098^{s} \pm 0.03^{s}$, Dec. $=-29^{\circ} 00^{\prime} 27.08^{\prime \prime} \pm 0.05$. Recent measurements in the near-infrared using large single dish telescopes or interferometers allowed to determine some of the parameters with greatly improved accuracy. This was a central aspect of the contribution given at the conference on 'Accretion Processes in Cosmic Sources II (APCS2018) that was held between 3 and 8 September, 2018, in Saint Petersburg (Russian Federation). Here, we give a brief summary of this aspect.

\section{Mass}

The mass is the most essential quantity to determine the nature of a black hole. Various probes have been used to constrain this parameter. The enclosed mass could be revealed through gaseous and stellar probes. This was first achieved by the observation of the $12.8 \mu \mathrm{m}$ NeII line emission originating in the Galactic Center mini-spiral by Wollmann et al. (1977). The obtained enclosed mass amounted to $4 \times 10^{6} \mathrm{M}_{\odot}$. This result could be confirmed and improved on by making use of stellar probes. Stellar radial velocity measurements of the stars in the Galactic Center stellar cluster were obtained by Krabbe et al. (1995). First stellar proper motions could be measured by Eckart \& Genzel $(1996,1997)$ and Ghez et al. (1998). Finally, first reliable accelerations (Ghez et al. 2000 and Eckart et al. 2002) and then orbit measurements of the star S2 (Schödel et al. 2002, Horrobin et al. 2004, Eisenhauer et al. 2003) gave consistent estimates of the enclosed mass. The early estimates of as low as $2.45 \pm 0.4 \times 10^{6} \mathrm{M}_{\odot}$ gradually converged to a higher value around $4.0 \pm 0.4 \times 10^{6} \mathrm{M}_{\odot}$. Using the stars closest to $\mathrm{SgrA}^{*}$, the mass estimate lies at a value around 4.0 million solar masses with an uncertainty of about 0.2 million solar masses (Boehle et al. 2016 and Parsa et al. 2017).

In Fig. 1 we show the development. The enclosed mass estimates obtained for the mini-spiral through gas measurements (Wollman et al. 1977) is marked with a bold face letter "G". Mass estimates derived from ensembles of stars in the central cluster using spectroscopy and proper motion date are shown by turquoise filled circles. They are labeled: 1: Krabbe et al. (1995); 2: Eckart et al. (1996); 3: Ghez et al. (1998); 4: Genzel et al. (2000). Mass estimates derived from orbital curvatures and stellar orbits are shown by red filled circles and labeled: 5: Eckart et al. (2002); 6: Schödel et al. (2002), Horrobin et al. (2004), Eisenhauer et al. (2003);7: Ghez et al. (2008); 8: Gillessen et al. (2009); 9: Do et al. (2013); 10: Boehle et al. (2016); 11: Parsa et al. (2017). 12: GRAVITY Collaboration (2018a). The most exact determination of the mass could be obtained recently by combining spectroscopic data with interferometric positional data obtained by the GRAVITY beam combiner instrument at the European Southern Observatory (ESO) using the Very Large Telescope Interferometry (VLTI) infrastructure (GRAVITY Collaboration 2018a). The corresponding mass values are listed in Tab. 1. 


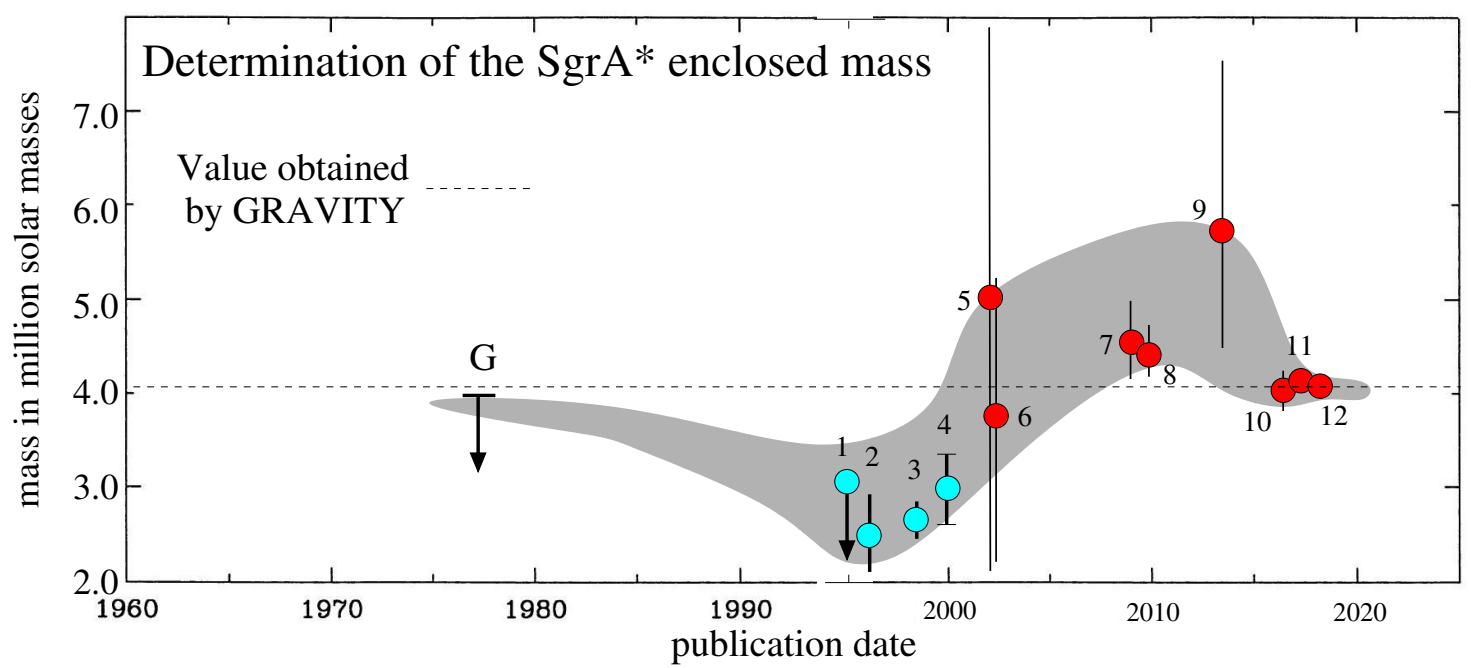

Figure 1: The enclosed mass of SgrA* at the Galactic Center in the course of four decades of measurements. The data are described in the text. The grey structure approximates the envelope of the measurements. The VLTI GRAVITY measurements were supported by VLT imaging and spectroscopy results as explained in GRAVITY Collaboration (2018a).

\section{Distance}

The coupling between the line of sight velocity and the transformation of proper motions measured in angular units to the motions in physical linear units is reflected in the fact that both the mass and distance are being determined at the same time. A classical method of determining the distance to the Galactic Center used globular clusters under the assumption that the Galactic Bulge is located at the center of the distribution (e.g., Shapley 1918, Racine \& Harris 1989, Francis et al. 2014). A more exact way is to make use of the stellar orbits deriving the mass and distance at the same time. In Fig. 2 and table Tab. 2 we use representative measurements to show the mass estimated as a function of publication date of the measurement. The most of the data presented by Genzel, Eisenhauer \& Gillessen (2010) and Reid (1993) are located in the zones labeled as $\alpha$ and $\beta$. The data used by Malkin et al. (2013) can mostly be found in zone $\gamma$. The calculated trends found by (Malkin et al. 2013) are represented by the solid and dashed lines in zone $\gamma$. Recent stellar orbit data are shown as turquoise filled circles and labeled: 1: Horrobin et al. (2004), Eisenhauer et al. (2003), Schödel et al. (2002); 2: Ghez et al. (2008); 3: Gillessen et al. (2009); 4: Ghez et al. (2008); 5: Do et al. (2013); 6: Majaess et al. (2009); 7: Vanhollebeke et al. (2009); 8: Do et al. (2013); 9: Dekany et al. (2013); 10: Francis et al. (2014); 11: Branham et al. (2014); 12: Boehle et al. (2016); 13: Parsa et al. (2017). 14: GRAVITY Collaboration (2018a).

Detailed astrometric and spectroscopic observations of the central high velocity stars have resulted in evermore accurate determinations of the distance to the central supermassive black hole SgrA* (Schödel et al. 2002, Eisenhauer et al. 2003, Horrobin et al. 2004, Ghez et al. 2008, Gillessen et al. 2009, Boehle et al. 2016). A combined orbital fit of the S2, S38 and S0-102 data, by Parsa et al. (2017) results in $M=4.15 \pm 0.13 \times 10^{6} M_{\odot}$ and $8.19 \pm 0.1 \mathrm{kpc}$. From Fig.2, we see that the initial measurements (as a function of time) started with high values of up to $10 \mathrm{kpc}$, however, by 
Table 1: Representative black hole mass estimates for SgrA*

\begin{tabular}{cll}
\hline \hline No. & references & mass in $10^{6} \mathrm{M}_{\odot}$ \\
\hline 1 & Krabbe et al. (1995) & 3 \\
2 & Eckart et al. (1996) & $2.45 \pm 0.4$ \\
3 & Ghez et al. (1998) & $2.6 \pm 0.2$ \\
4 & Genzel et al. (2000) & $2.6-3.3$ \\
5 & Eckart et al. (2002) & $5 \pm 3$ \\
6 & Schödel et al. (2002), Horrobin et al. (2004), & \\
& Eisenhauer et al. (2003) & $3.7 \pm 1.5$ \\
7 & Ghez et al. (2008) & $4.5 \pm 0.4$ \\
8 & Gillessen et al. (2009) & $4.30 \pm 0.4$ \\
9 & Do et al. (2013) & $5.76_{-1.26}^{+1.76}$ \\
10 & Boehle et al. (2016) & $4.0 \pm 0.2$ \\
11 & Parsa et al. (2017) & $4.15 \pm 0.13$ \\
12 & GRAVITY Collaboration (2018a) & $4.10 \pm 0.03$ \\
\hline \hline
\end{tabular}

now the values have converged to a combined mass and distance of $M=4.10 \pm 0.03 \times 10^{6} M_{\odot}$ and $8.12 \pm 0.03 \mathrm{kpc}$, with unprecedented small uncertainty thanks to the high precision measurements of the GRAVITY instrument (GRAVITY Collaboration 2018a).

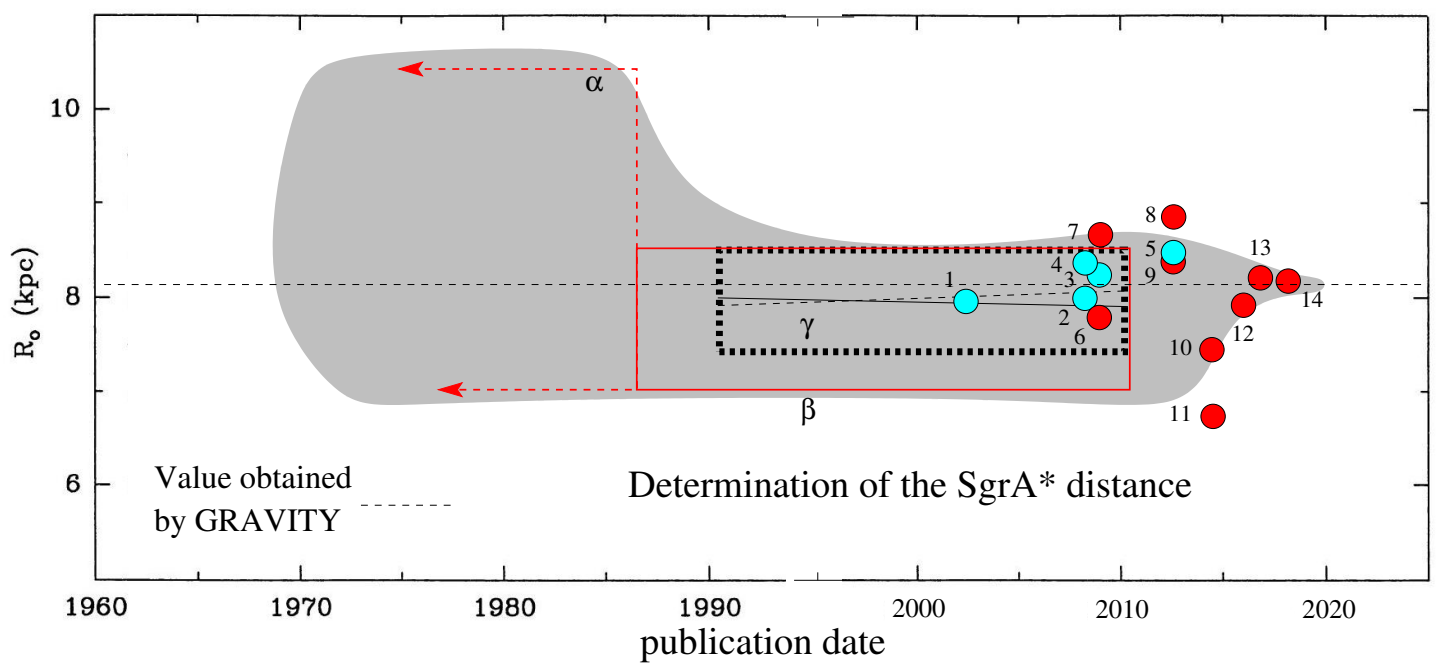

Figure 2: Distance estimate for the Center of the Milky Way. The data are described in the text. The grey structure approximates the envelope of the measurements.

\section{Spin}

The SgrA* supermassive black hole has usually been described within the framework of classical General Relativity, namely, its Kerr metric solution. For black holes the spin parameter $a$ can 
Table 2: Representative distance estimates for SgrA*

\begin{tabular}{cll}
\hline \hline No. & references & distance in kpc \\
\hline 1 & Horrobin et al. (2004), Eisenhauer et al. (2003) & \\
& Schödel et al. (2002); & $7.94 \pm 0.42$ \\
2 & Ghez et al. (2008); & $8.0 \pm 0.6$ \\
3 & Gillessen et al. (2009a); & $8.28 \pm 0.32$ \\
4 & Ghez et al. (2008); & $8.46 \pm 0.40$ \\
5 & Do et al. (2013); & $8.46 \pm 0.40$ \\
6 & Majaess et al. (2009) & $7.7 \pm 0.7$ \\
7 & Vanhollebeke et al. (2009) & $8.7 \pm 0.71$ \\
8 & Do et al. (2013) & $8.92 \pm 0.56$ \\
9 & Dekany et al. (2013) & $8.33 \pm 0.15$ \\
10 & Francis et al. (2014) & $7.4 \pm 0.28$ \\
11 & Branham et al. (2014) & $6.72 \pm 0.39$ \\
12 & Boehle et al. (2016) & $7.9 \pm 0.2$ \\
13 & Parsa et al. (2017) & $8.19 \pm 0.1$ \\
14 & GRAVITY Collaboration (2018a) & $8.12 \pm 0.03$ \\
\hline \hline
\end{tabular}

be obtained via the angular momentum parameter $a=J / M c$. Here, $J$ is the angular momentum and $M$ the black hole mass. Following this definition one can define a dimensionless parameter $a^{*}=a c^{2} / G M$, which lies between 0 and 1 . A non-rotating black hole is described by $a^{*}=0$ and maximally rotation black hole has a spin parameter of $a^{*}=1$. The spin cannot be measured directly but must be derived by modeling quantities that correlate with the spin value, e.g., light curves and polarization behavior, if one assumes the presence of orbiting hot-spots.

In comparison to an orbiting spot model one finds that the variability indicates a spin parameter of $a=0.5$ and inclined orbits (Shcherbakov et al. 2012, Vincent et al. 2011, Broderick \& Loeb 2006, Trippe et al. 2007). Higher inclinations ( $45^{\circ}$ and larger) are suggested by the light curve analyses of Meyer et al. (2006) and Karssen et al. (2017). The motion of orbiting hot-spots during flares of SgrA* has recently been measured using the GRAVITY instrument (GRAVITY Collaboration 2018b). In this case lower inclinations, i.e., $\leq 45^{\circ}$ and less are indicated. This may suggest that an orbital plane through which accretion into the inclined orbits and finally onto SgrA* occurs is not well defined.

In Tab. 4 we list various model dependent estimates of the black hole spin for the supermassive back hole SgrA*. For the estimates from the GRAVITY measurements (Fig.3, GRAVITY Collaboration 2018b) the remarks are that 1) all three measurements during a flare are consistent with scenarii between $a=+1$ and $a=0$, and 2) at least one, possibly two of the measurements could also be consistent with scenarii between $a=0$ and $a=-1$. The zone $\zeta$ would harbor spots that are too distant from the black hole for the measured period, or have too slow a period for their given distance. In zone $\xi$ one may find spots that are too close to the black hole for the measured period, or have too high a period for their given distance. Such a situation may occur if additional 
Table 3: Representative model dependent spin estimates for SgrA*

\begin{tabular}{clll}
\hline \hline No. & spin & method & reference \\
\hline 1 & $0.52 \pm 0.13$ & NIR variab. & Genzel et al. 2003 \\
2 & 0.5 & NIR variab. & Eckart et al. 2006 \\
3 & $>0.4$ & NIR variab. & Meyer et al. 2006a \\
4 & $>0.5$ & NIR variab. & Meyer et al. 2006b, 2007 \\
5 & 0.92 & NIR variab. & Witzel et al. 2018 \\
6 & $\mathrm{a}=0 \ldots 1^{1)}$ & NIR interfer. & GRAVITY Collab. 2018b \\
7 & $\mathrm{a}=-1 \ldots 0^{2)}$ & NIR interfer. & GRAVITY Collab. 2018b \\
& & & \\
8 & $<0.8$ & radio variab. & Broderick et al. 2011 \\
& & & \\
9 & $0.9959 \pm 0.0005$ & X-ray data & Aschenbach et al. 2010 \\
10 & $>0.22$ & X-ray data & Belanger et al. 2006 \\
\hline \hline
\end{tabular}

Table 4: We list representative estimates of the dimensionless spin parameter $a^{*}=a c^{2} / G M$. The spin estimates were derived from near-infrared or radio variability data, infrared interferometry data, or X-ray imaging data. With respect to the NIR interferometry data a stationary black hole or even a scenario in with the orbiting matter is on a retrograde orbit cannot be excluded (GRAVITY Collaboration 2018b). Remarks 1) an 2) are explained in the text.

forces influence the orbit of the spots. In some cases this may be established by a high degree of magnetic coupling or viscosity within the putative disk which these spots may be part of (Mondal \& Mukhopadhyay 2018, and Dewberry, Latter \& Ogilvie 2019). This is also demonstrated in Fig.4 in which we show the possible spin $a$ as a function of the orbiting spot radial distances.

\section{Charge}

Following the infamous 'no-hair' theorem, in addition to mass and spin, the charge is another essential parameter that characterizes a black hole (Israel 1968, Carter 1971). In most cases one assumes the charge becomes quickly neutralized, and the black hole charge is thus set to zero (however, see Kovar et al. 2011, Cremaschini et al. 2013). There are, however, processes that can lead to the formation of non-zero electric charge of black holes. In the case of SgrA* this can potentially be measured (Zajacek et al. 2018a, 2018b). The fact that there is a difference in thermal velocities of electrons and protons in a fully ionized plasma that surrounds SgrA* results in a classical argument to get a small net black hole charge. As a transient phenomenon over viscous time-scale Zajacek et al. (2018a, 2018b) find an upper limit of about $10^{9} \mathrm{C}$. Due to the frame dragging effect and the twisting of magnetic field lines located close to the event horizon a black hole may also induce a net electric charge. Strong magnetic field may be present in the vicinity of a rotation super massive black hole SgrA* (Eatough et al. 2013, Morris 2015, GRAVITY Collaboration 2018b, Eckart 2006, 2012) with the strength of about 10 Gauss. The induced charge that is implied is $\leq 10^{15} \mathrm{C}$. 


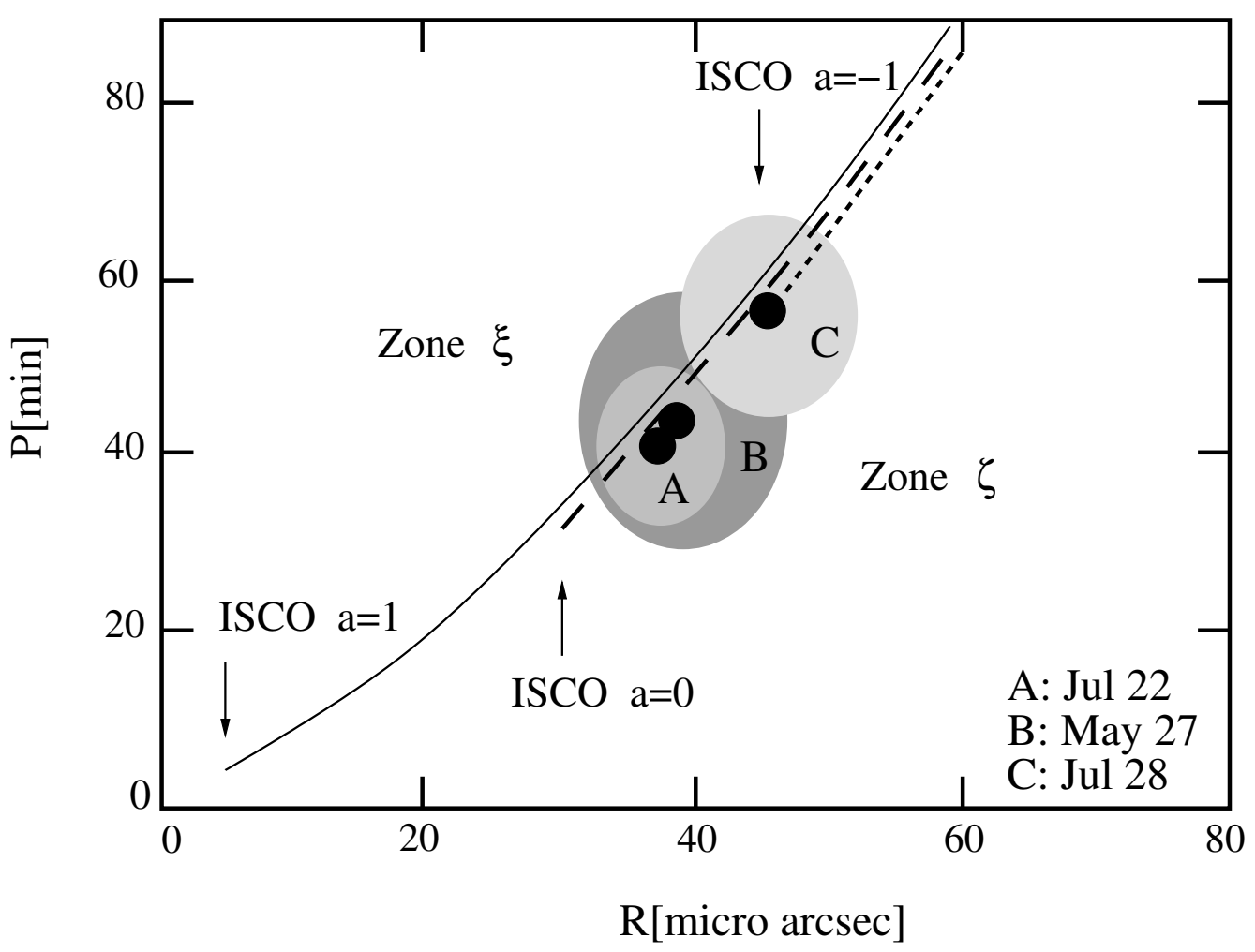

Figure 3: Period vs. spot radius diagram showing where the GRAVITY measurements come to lie (based on GRAVITY Collaboration 2018b). The relevance of zones $\zeta$ and $\xi$ are explained in the text. The arrows point at the stating points of the $a=-1,0,1$ tracks.

These charge values only have a very minor effect on the space time metric. Therefore, a Kerr metric is still a very good approximation to describe the black hole. However, the motion of charged particles may be influenced. Zajacek et al. (2018a, 2018b) argue that a small charge of SgrA* is probably positive with an upper limit $10^{15} \mathrm{C}$. The authors also present observational tests to estimate the charge of SgrA*. This can be achieved via measuring a flattening and decrease in the thermal bremsstrahlung surface brightness profile inside a region that approximately coincides with the location of the cluster of high velocity S-stars. Using recent X-ray observations of the Chandra X-ray observatory Zajacek et al. (2018a, 2018b) can already now put an observational upper limit on the SgrA* charge of $\leq 3 \times 10^{8} \mathrm{C}$. Hence, the charge is only very small and most likely transient and neutralized but reoccurring over a viscous time-scale.

\subsection{Orientation}

To obtain information on the orientation of the spinning black hole SgrA* is largely model dependent procedure, however, here are a few observational facts that are valuable in this regard. A fundamental assumption is that the matter in the immediate surroundings of the black hole is indeed 


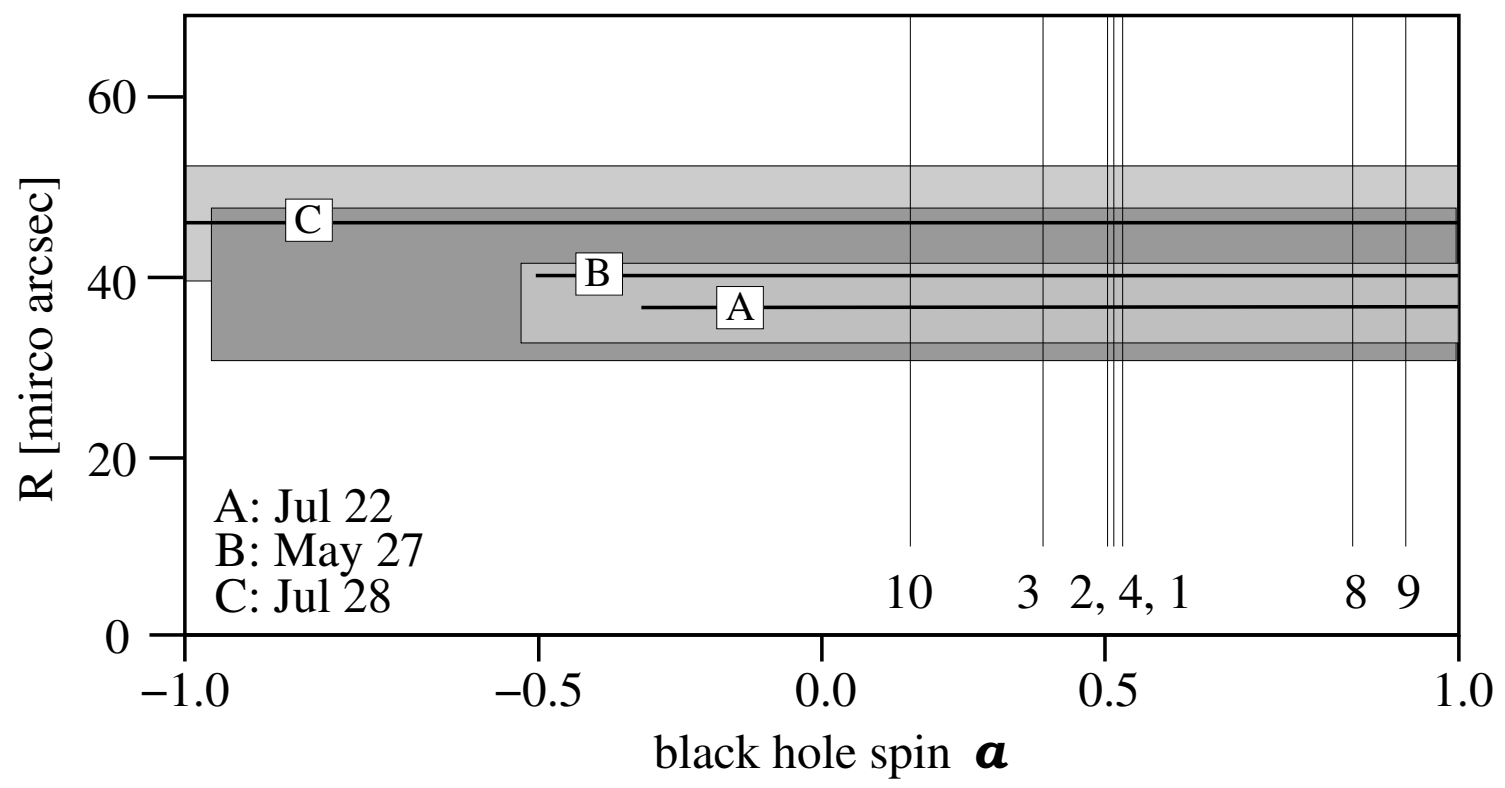

Figure 4: Possible spins $a$ as a function of the orbiting spot radial distances. The dates of GRAVITY observations are given (based on GRAVITY Collaboration 2018b). The number labels for the individual spin measurements refer to the numbering in Tab. 3. The bold face lines labeled with the observing data A, $\mathrm{B}$ or $\mathrm{C}$ correspond to the central value, the grey shaded areas include the uncertainties.

coupled to its spin. The hope is also that there is something like a mid-plane of the accretion stream, i.e., a preferred orbital plane. In that case we can interpret information on this matter to derive its geometrical orientation and can then claim that it agrees with that of the black hole.

Important facts are here the presence of a preferred angle of the E-vector of the polarized light from SgrA* in the optically thin infrared domain (Shahzamanian et al. 2015). If there is a disk or jet like arrangement, this will be reflected in the preferred polarization angle of about $13^{\circ} \pm 15^{\circ}$ (i.e. east of north). The polarized light curves can be modeled with a relativistic disk or hot-spot system. This gives model dependent information concerning the orientation of the orbiting matter. Such a result was obtained by Meyer et al. (2007). Here the authors find that the position angle of the angle which is normal to the equatorial plane of the orbit is in the range of $60^{\circ}-108^{\circ}$ (east of north). This is in agreement with findings by Zamaninasab et al. (2010). Meyer et al. (2007), however, find a relatively large value for the inclination angle well above $45^{\circ}$. The new GRAVITY measurements (GRAVITY Collaboration 2018b) indicate that there are indeed flux entities in orbit around SgrA*. Given that the inclination found by the GRAVITY experiment are rather of the order of - or lower than $45^{\circ}$ and the overall orientation of the orbits in the sky is not very well determined.

However, as mentioned in the beginning of the paragraph it is not clear how stable (or variable in time) a mid-plane of the accretion stream is. The essence is that the model dependent interpretations of the observational facts indicate that the spin vector of the SgrA* system is oriented in the direction east or north and is pointing towards us at some appreciable angle. 


\section{Summary}

Due to its proximity, $\mathrm{SgrA}^{*}$ at the center of the Milky Way is best suited to determine its mass and distance, spin, and charge.

Given the precise measurements of the GRAVITY interferometer at the VLTI facility the high precision of the infrared spectroscopy measurements and the high angular resolution X-ray imaging one can summarize: For the distance and mass of SgrA* one finds $R_{S g r A *}=8.12 \pm 0.03 \mathrm{kpc}$ and $M_{S g r A *}=(4.10 \pm 0.03) \times 10^{6} \mathrm{M}_{\odot}$. The spin is model dependent and currently less well determined. There are indications that it is between $a=0.5$ and $a=0.92$. For the charge we can give an observational upper limit as $Q_{S g r A *} \leq 3 \times 10^{8} \mathrm{C}$. Repeated infrared interferometric measurements, improved spectroscopy and future higher angular resolution measurements will help to improve these results significantly. The detection of hot spots by GRAVITY may indicate that a more precise spin determination will also be possible.

\section{References}

[1] Aschenbach, B., 2010, MmSAI 81, 319

[2] Belanger, G.; Terrier, R.; de Jager, O. C.; Goldwurm, A.; et al., 2006, JPhCS 54, 420

[3] Boehle A., Ghez A.M., Schödel R., Meyer L., 2016, ApJ 830, 17

[4] Branham R.L., 2014, Ap\&SS 353, 179

[5] Broderick, A.E.; Loeb, A., 2006, MNRAS 367, 905

[6] Broderick, A.E.; Fish, V.L.; Doeleman, S.S.; Loeb, A., 2011, ApJ 735, 110

[7] Carter, B., 1971, Phys. Rev. Lett. 26, 331

[8] Cremaschini, C., et al., 2013, ApJSS 209, id. 15

[9] Dekany, I.; Minniti, D.; Catelan, M.; et al.; 2013, ApJ 776, L19

[10] Dewberry, J.W.; Latter, H.N.; Ogilvie, G.I.; 2019, MNRAS 483, 1609

[11] Do, T.; Martinez, G. D.; Yelda, S.; Ghez, A.; et al., 2013, ApJ 779, L6

[12] Eatough, R.P.; Falcke, H.; Karuppusamy, R.; Lee, K. J.; , 2013,Nature 501, 391

[13] Eckart A., Genzel R., 1996, Nature 383, 415

[14] Eckart A., Genzel R., 1997, MNRAS 284,576

[15] Eckart A., Genzel R., Ott T, Schödel R., 2002, MNRAS 331, 917

[16] Eckart, A.; Schödel, R.; Meyer, L.; et al., 2006, A\&A 455, 1

[17] Eckart, A.; Garcia-Marin, M.; Vogel, S. N.; et al., 2012, A\&A 537, 52

[18] Eckart, A.; Hüttemann, A.; Kiefer, C.; Britzen, S.; Zajacek, M.; Lämmerzahl, C.; 2017, Foundations of Physics 47, 553

[19] Eisenhauer F., Schödel R., et al., 2003, ApJL 597, L121

[20] Francis C., Anderson E., 2014, MNRAS 441, 1105

[21] Genzel, R.; Pichon, C.; Eckart, A.; Gerhard, O. E.; Ott, T., 2000, MNRAS 317, 348 
[22] Genzel, R.; Schödel, R.; Ott, T.; Eckart, A.; 2003, Nature 425, 934

[23] Genzel, R.; Eisenhauer, F.; Gillessen, S., 2010, RvMP 82, 3121

[24] Ghez A.M., Klein B.L., Morris M., Becklin E.E., 1998, ApJ 509, 678

[25] Ghez, A. M.; Morris, M.; Becklin, E. E.; et al., 2000, Nature 407, 349

[26] Ghez, A.M.; Salim, S.; Weinberg, N.N.; et al., 2008, ApJ 689, 1044

[27] Gillessen, S.; Eisenhauer, F.; Fritz, T. K.; et al., 2009, ApJ 707, L114

[28] Gravity Collaboration; Abuter, R.; Amorim, A.; et al., 2018a, A\&A 615, L15

[29] Gravity Collaboration; Abuter, R.; Amorim, A.; et al., 2018b, A\&A 618, L10

[30] Horrobin M, Eisenhauer F, et al., 2004, Astron.Nachr. 325, 88

[31] Israel, W., 1968, Commun. Math. Phys. 8, 245

[32] Karssen, G. D.; Bursa, M.; Eckart, A.; et al., 2017, MNRAS 472, 4422

[33] Kovar, J., et al., 2011, Phys. Rev D 84, 084002

[34] Krabbe A., Genzel R., Eckart A., et al., 1995, ApJL 447, L95

[35] Majaess D.J., Turner D.G., Lane D.J., 2009, MNRAS 398, 263

[36] Malkin Z.M., 2013, Astronomy Reports 57, 128

[37] Menten K.M., Reid M.J., Eckart A., Genzel R., 1997, ApJL 475, L111

[38] Meyer, L.; Eckart, A.; Schödel, R.; et al., 2006, A\&A 460, 15

[39] Meyer, L.; Schödel, R.; Eckart, A.; et al. 2006, A\&A 458, L25

[40] Meyer, L.; Schödel, R.; Eckart, A.; 2007, A\&A 473, 707

[41] Mondal, T.; Mukhopadhyay, B.; 2018, Cosp 42, 2333

[42] Morris, M.R., 2015, Lessons from the Local Group: A Conference in honour of David Block and Bruce Elmegreen, ISBN 978-3-319-10613-7. Springer Intern. Publ. Switzerland, 2015, p. 391

[43] Parsa, M.; Eckart, A.; Shahzamanian, B.; Karas, V.; Zajaek, M.; et al., 2017, ApJ 845, 22

[44] Racine, R.; Harris, W.E.; 1989, AJ 98, 1609

[45] Reid M.J., Menten K.M., Genzel R., Ott T., Schödel R., Eckart A., 2003, ApJ 587, 208

[46] Reid M.J., 1993, ARA\&A 31, 345

[47] Schödel R., Ott T., et al.; 2002, Nature 419, 694

[48] Shahzamanian, B.; Eckart, A.; Valencia-S., M.; Witzel, G.; et al., 2015, A\&A 576, 20

[49] Shapley, H., 1918, PASP 30, 42

[50] Shcherbakov, R.V.; Penna, R.F.; McKinney, J.C., 2012, ApJ 755, $133 \mathrm{~S}$

[51] Trippe, S.; Paumard, T.; Ott, T.; et al., 2007, MNRAS 375, 764

[52] Zajacek, M.; Tursunov, A.; Eckart, A.; Britzen, S., 2018a, MNRAS 480, 4408

[53] Zajacek, M.; Tursunov, A.; Eckart, A.; Britzen, S.; 2018b, in Conf. Proc. of the FISICPAC-2018 conference (University of Sharjah, UAE), Journal of Physics: Conference Series, 2018arXiv181203574Z 
[54] Zamaninasab, M.; Eckart, A.; Witzel, G.; Dovciak, M.; Karas, V.; et al., 2010, A\&A 510, 3

[55] Vanhollebeke E., Groenewegen M.A.T., Girardi L., 2009, A\&A 498, 95

[56] Vincent, F. H.; Paumard, T.; Perrin, G.; et al.; 2011, MNRAS 412, 2653

[57] Witzel, G.; Martinez, G.; Hora, J.; et al., 2018, ApJ 863, 15

[58] Wollman E.R., Geballe T.R., Lacy J.H., Townes C.H., Rank D.M., 1977, ApJL 218, L103 